\title{
Implementation of the Coordination Equation for Determining the Transport-Related Losses in Economic Dispatch Phenomena
}

\section{Arief Goeritno}

Electrical Engineering Study Program, Universitas Ibn Khaldun Bogor; Jl. K.H. Sholeh Iskandar km.2, Kedung Badak, Tanah Sareal, Bogor 16164, West Java, Indonesia

Corresponding Author Email: arief.goeritno@uika-bogor.ac.id

https://doi.org/10.18280/mmep.070216

Received: 14 February 2020

Accepted: 16 May 2020

\section{Keywords:}

coordination equation, economic dispatch phenomena, the power loss on transmission line

\begin{abstract}
In this manuscript explains the classical aspects of economic dispatch phenomena through a theoretical approach. The method for minimizing the generation cost of the thermal generating unit will fail, if without covering the power losses on the transmission line, while the fuel cost is the largest component in the thermal generating unit. Based on that, the cost of producing the electrical power from a thermal generator should be sought as economically as possible. The existence of an incremental fuel cost of one generating unit may be lower than that of another, but the plant is located away from the load center which affects the power loss of transmission line has the large value. Optimization of fuel costs and power loss of transmission line are used the coordination equation for completion. In the coordination equation, economic costs are generated and achieved, when the total incremental cost of fuel that multiplied by the penalty factor is same for all generators. To calculate the achievement of economic dispatching value in electric power system with type of thermal generation with calculation of power loss of transmission line and value of limitation of generation of generating unit as generating function, hence can be reached by some way of calculation.
\end{abstract}

\section{INTRODUCTION}

The ability of the power supply to the load as a determinant of the reliability of the electrical power system, so always strived that the power generated must equal the demand on the load side and the power loss on the transmission line for the condition at any time [1-5]. The fuel cost at the thermal generating station for the power plant is a function of load power $[1,4,5]$. Increasing the power for supply to the load on a fossil fuel thermal power plant unit is a driver of increasing the quantity of fuel per unit time which ultimately drives the increase in cost per unit time [1, 5, 6-9]. The fluctuation of demands on the load side as a fluctuator of the associated fuel costs, so as to obtain a pattern of correlation of both $[2,4]$. A waiver of power losses on the transmission line affects failure in cost suppression $[1,3]$.

Determining the distribution of the load economically between the generating units, consideration of losses on the transmission line as a generation function is required [1]. The presence of additional fuel costs on one busbar generating unit may be higher than the system's additional cost, then the option remains to the additional cost of the system, in order for the electric power to move deep into the load center [5, 7]. The power loss on the transmission line from the generating unit which has a lower additional cost may be too great, so in terms of economics it is necessary to decrease the load on the generating unit at a lower additional cost, and increase power to the generating unit at a higher additional cost [1, 3-5, 7-9].

The determination of power losses on transmission line using the coordination equation when economic dispatch in electrical power system with case of network structure consisting of four buses with two generating buses and two load buses, while the value of generating limits from the thermal generation station is taken into calculation. The economic dispatch phenomena are an initial condition for the existence of an optimum power flow, because a specific problem [10], so it becomes a determining factor for the success of the optimum power flow condition [11].

Limitations of economic dispatch is a condition caused by (i) generating units and loads are not all connected to the same bus and (ii) the economic dispatch may result in unacceptable flows or voltages in the network [12]. The real power losses minimization is required objective function for minimizing active power loss which can be formulated [13]. The transmission system has several constraints which can be categorized in the form of equality and inequality constraint [13]. However, adding inequality constraints for each problem is not practical in more complex situations, so that needed a more general approach [12]. In this manuscript explains the classical aspects of economic dispatch phenomena through a theoretical approach.

\section{MATERIAL AND METHOD}

\subsection{Material}

A network equivalent of a single-node system with all the generators and the load is directly connected, it can be said to be a representation of conditions with neglected the power loss 
on transmission line, so the penalty factor for each plant becomes one [3]. When the power loss on transmission line is considered, then the economic dispatch of electric power must be through iterative process with a non-linear coordination equation solving [3]. Deriviting the equation of power loss on transmission line in an electrical energy system, it should refer to a simple system consisting of two generators and two loads with a transmission network represented by a bus impedance matrix [2]. Decreasing the power loss equation is done through in two stages. The first stage used invariant power transformation to the system bus to show the system loss only on the generator current. The second stage transforms the generator current into the generating power output going to the form of the power loss equations for the system with the source [2].

The start of the formulation taken with reference to bus-1 and bus- 2 is the generating bus, bus- 3 and bus- 4 are load bus, and the $n$-th bus is a neutral system. Schematic diagram of simple system analogy of electrical power with four buses [2], as shown in Figure 1.

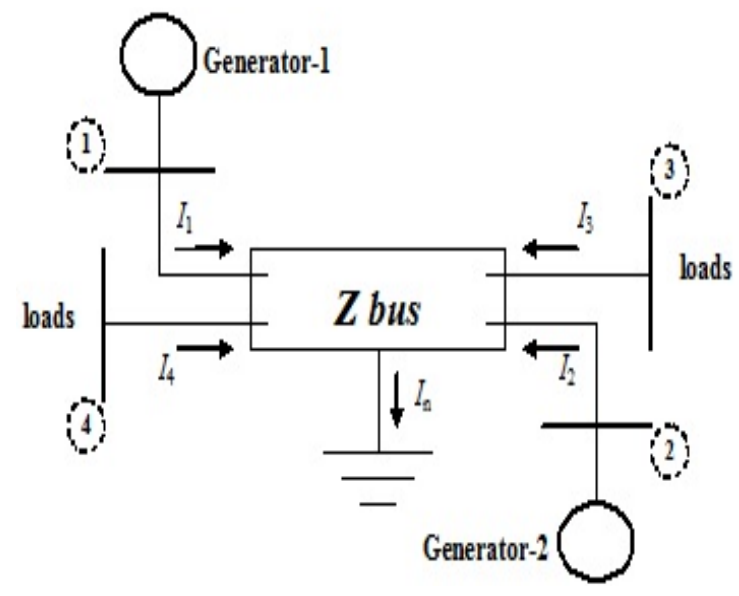

Figure 1. Schematic diagram of simple system analogy of electrical power with four buses

The currents of $I_{3}$ and $I_{4}$ injected at the load bus are combined simultaneously for the formation of the system load bus [2], to obtain the Eq. (1).

$$
I_{3}+I_{4}=I_{D}
$$

It is assumed, each load with a constant and balanced division of the total load [2], so that it is obtained as Eq. (2).

$$
I_{3}=d_{3} \cdot I_{D} \text { and } I_{4}=d_{4} \cdot I_{D}
$$

then:

$$
d_{3}+d_{4}=1
$$

\subsection{Method}

The problem of minimizing the overall cost function in an interconnected system must be with consideration the power loss on transmission line, so that the power balance [4] is defined as Eq. (4).

$$
\left(\sum_{i=1}^{k} P_{G i}\right)-P_{\text {Losses }}-P_{\text {Demand }}=0
$$

Substituting the transmission line power losses into the system optimization, the Lagrange function $[3,4]$ is obtained as Eq. (5).

$$
L F=C_{i}\left(P_{G i}\right)-\lambda \cdot\left[\left(\sum_{i=1}^{k} P_{G i}\right)-P_{\text {Losses }}-P_{\text {Demand }}\right]
$$

For optimum real power distribution and incremental cost are achieved when conditions such as Eqns. (6) and (7) is met $[1,4]$.

$$
\begin{gathered}
\frac{\delta}{\delta P_{G i}} L F=0 \\
\frac{\delta}{\delta \lambda} L F=0
\end{gathered}
$$

where, $i=1,2, \ldots, k$.

Solution to Eq. (6) is obtained the result of Eq. (8).

$$
\frac{\delta}{\delta P_{G i}} C_{i}-\lambda \cdot \frac{\delta}{\delta P_{G i}}\left[P_{G i}-P_{\text {Losses }}-P_{\text {Demand }}\right]=0
$$

while solution to Eq. (7) is obtained the result of Eq. (9).

$$
-\frac{\delta}{\delta \lambda}\left[P_{G i}-P_{\text {Losses }}-P_{\text {Demand }}\right]=0
$$

One generator's output change only affects the cost of the generator, so through the rearrangement of Eq. (8), we get the result as Eq. (10) or (11) or (12) [3, 4].

$$
\begin{gathered}
\frac{\delta}{\delta P_{G i}} C_{i}-\lambda \cdot\left[1-\frac{\delta}{\delta P_{G i}} P_{\text {Losses }}\right]=0 \\
\frac{\delta}{\delta P_{G i}} C_{i} \cdot\left[\frac{1}{1-\frac{\delta}{\delta P_{G i}} P_{\text {Losses }}}\right]=\lambda \\
\frac{\delta}{\delta P_{G i}} C_{i} \cdot \lambda_{i}=\lambda
\end{gathered}
$$

for all values of the $i$-th $\left(P_{G i}^{\min .} \leq P_{G i} \leq P_{G i}^{\max }\right)$ [3], where $\lambda_{i}=$ $\frac{1}{1-\frac{\delta}{\delta P_{G i}} P_{\text {Losses }}}$ is called the $i$-th bus penalty factor [3, 4]. Eq. (12) is called as a coordination Eq. [3], since the incremental costs and transmission line losses are coordinated, in order to achieve the most optimum results. Partial differentiation of $\frac{\delta}{\delta P_{G i}} P_{\text {Losses }}$ is called as incremental transmission losses or power loss in the auxiliary transmission line at the $i$-th throttling unit [3]. The use of Eq. (6), generated the value of the Eq. (4) as a normal active balance equilibrium equation [3].

The cost of a penalty factor is the same for all generators or written as Eq. (13).

$$
\frac{d}{d P_{G 1}} C_{1} \cdot \lambda_{1}=\frac{d}{d P_{G i 2}} C_{2} \cdot \lambda_{2}=K=\frac{d}{d P_{G k}} C_{k} \cdot \lambda_{k}=\lambda
$$

For small changes to the system load, for example $\Delta P_{\text {Demand }}$, the generation of power in the $k$-th generating unit changes by $\Delta P_{G k}$. The power output from the other generator 
is kept constant, then the change in the power loss on transmission line is equal to $\Delta P_{\text {Losses }}$, so that $\Delta P_{G k}-$ $\Delta P_{\text {Losses }}=\Delta P_{\text {Demand }}, \quad$ and $\quad \frac{d}{d P_{G k}} C_{k} \cdot \lambda_{n}=\frac{d}{d P_{G k}} C_{k}$. $\frac{1}{1-\frac{\Delta}{\Delta P_{G i}} P_{\text {Losses }}}$. The existence of each generating unit with fuel cost characteristics as a second-order equation, then used for easy understanding to $\lambda$ (incremental cost) of the fuel [3].

\section{RESULTS AND DISCUSSIONS}

Can be simplified on a simple system consisting of 2 (two) units of generating units, so that the value of $\lambda$ for each generating unit, such as Eq. (14).

$$
\lambda_{1}=\frac{d}{d P_{G 1}} C_{1}=a_{1} \cdot P_{G 1}+b_{1}
$$

and

$$
\lambda_{2}=\frac{d}{d P_{G 2}} C_{2}=a_{2} \cdot P_{G 2}+b_{1}
$$

Settlement for $P_{G 1}$ and $P_{G 2}$ are obtained as Eq. (15).

$$
P_{G 1}=\frac{\lambda-b_{1}}{a_{1}} \text { and } P_{G 2}=\frac{\lambda-b_{2}}{a_{2}}
$$

and the settlement of $\lambda$ value is obtained as Eq. (16).

$$
\begin{gathered}
\lambda=\left(\sum_{i=1}^{2} \frac{1}{a_{1}}\right)^{-1}\left(P_{G 1}+P_{G 2}\right)+\left(\sum_{i=1}^{2} \frac{1}{a_{1}}\right)^{-1} \\
\cdot\left(\sum_{i=1}^{2} \frac{b_{1}}{a_{1}}\right)^{-1}
\end{gathered}
$$

In another form the Eq. (16) is written as Eq. (17).

$$
\lambda=a_{T} \cdot P_{G T}+b_{T}
$$

where:

$$
\begin{gathered}
a_{T}=\left(\sum_{i=1}^{2} \frac{1}{a_{1}}\right)^{-1} ; P_{G T}=P_{G 1}+P_{G 2} ; \text { and } \\
b_{T}=a_{T} \cdot\left(\sum_{i=1}^{2} \frac{b_{1}}{a_{1}}\right)^{-1} .
\end{gathered}
$$

Implementation of the system with the generating unit, then the coefficient of Eq. (17) changes into Eqns. (18) and (19).

$$
\begin{gathered}
a_{T}=\left(\sum_{i=1}^{2} \frac{1}{a_{1}}\right)^{-1}=\left(\frac{1}{a_{1}}+\frac{1}{a_{2}}+\cdots+\frac{1}{a_{k}}\right)^{-1} \\
b_{T}=a_{T} \cdot\left(\sum_{i=1}^{2} \frac{b_{1}}{a_{1}}\right)^{-1}=a_{T} \cdot\left(\frac{b_{1}}{a_{1}}+\frac{b_{2}}{a_{2}}+\cdots+\frac{b_{k}}{a_{k}}\right)^{-1}
\end{gathered}
$$

and the total output of the generating unit $P_{G T}=P_{G 1}+P_{G 2}+\ldots$ $+P_{G K}$

The substitution of the coordination equation by taking into account of the equations for power loss on the transmission line, then for a simple system consisting of 2 (two) generating plants is obtained as Eq. (20).

$$
\begin{aligned}
\left(a_{1} \cdot P_{G 1}+b_{1}\right)- & \lambda \\
& +\left(2 \cdot L_{11} \cdot P_{G 1}+2 \cdot L_{12} \cdot P_{G 2}\right. \\
& \left.+L_{10}\right)
\end{aligned}
$$

Through the reassembling of its tribes and dividing the resultant equation by $\lambda$, giving as Eqns. (21) and (22).

$$
\begin{gathered}
\left(\frac{a_{1}}{\lambda}+2 \cdot L_{11}\right) \cdot P_{G 1}++2 \cdot L_{12} \cdot P_{G 2} \\
=\left(1-L_{10}\right)-\frac{b_{1}}{\lambda} \\
2 \cdot L_{21} \cdot P_{G 1}+\left(\frac{a_{2}}{\lambda}+2 \cdot L_{22}\right) P_{G 2}=\left(1-L_{20}\right)-\frac{b_{2}}{\lambda}
\end{gathered}
$$

In the matrix form for the two units, it is obtained as Eq. (23).

$$
\begin{array}{rc}
{\left[\begin{array}{cc}
\left(\frac{a_{1}}{\lambda}+2 \cdot L_{11}\right) & 2 \cdot B_{12} \\
2 \cdot L_{21} & \left(\frac{a_{2}}{\lambda}+2 \cdot L_{22}\right)
\end{array}\right] \cdot\left[\begin{array}{l}
P_{G 1} \\
P_{G 2}
\end{array}\right]} \\
=\left[\begin{array}{c}
\left(1-B_{10}\right)-\frac{b_{1}}{\lambda} \\
\left(1-B_{20}\right)-\frac{b_{2}}{\lambda}
\end{array}\right]
\end{array}
$$
(24).

$$
\begin{gathered}
\left(\frac{a_{1}}{\lambda}+2 \cdot L_{11}\right) \cdot P_{G 1}+\sum_{\substack{i=1 \\
j \neq 1}}^{k} 2 \cdot L_{i j} \cdot P_{G j}=\left(1-L_{10}\right) \\
-\frac{b_{1}}{\lambda}
\end{gathered}
$$

and in matrix form as Eq. (25).

$$
\begin{aligned}
& {\left[\begin{array}{ccccc}
\left(\frac{a_{1}}{\lambda}+2 \cdot L_{11}\right) & 2 \cdot L_{12} & & \cdots & 2 \cdot L_{1 k} \\
2 \cdot L_{21} & \left(\frac{a_{1}}{\lambda}+2 \cdot L_{22}\right) & & \cdots & 2 \cdot L_{2 k} \\
\vdots & \vdots & \ddots & & \vdots \\
2 \cdot L_{k 1} & 2 \cdot L_{k 2} & \cdots & \left(\frac{a_{1}}{\lambda}+2 \cdot L_{k k}\right)
\end{array}\right]\left[\begin{array}{c}
P_{G 1} \\
P_{G 2} \\
\vdots \\
P_{G k}
\end{array}\right]=} \\
& {\left[\begin{array}{c}
\left(1-L_{10}\right)-\frac{b_{1}}{\lambda} \\
\left(1-L_{20}\right)-\frac{b_{2}}{\lambda} \\
\vdots \\
\left(1-L_{k 0}\right)-\frac{b_{k}}{\lambda}
\end{array}\right]}
\end{aligned}
$$

Taking into account the balance of power, the equation for power loss on the transmission line become as Eq. (26).

$$
\begin{gathered}
P_{\text {Losses }}=\sum_{i=1}^{2} \sum_{j=1}^{2} P_{G i} \cdot L_{i j} \cdot P_{G j}+\sum_{i=j}^{2} L_{10} \cdot P_{G i}+L_{00} \\
+P_{\text {Demand }}-\sum_{i=1}^{k} P_{G i}=0
\end{gathered}
$$

The constraint function in optimization refers to Eq. (4), where,

$$
\begin{gathered}
P_{G i}^{\min } \leq P_{G i}<P_{G i}^{\max } \quad i=1,2, \cdots, k \\
P_{G i}^{\text {max. }} \geq P_{\text {Demand }} \quad i=1,2, \cdots, k
\end{gathered}
$$


Based on Figure 1, it is further analyzed by selection of the $\mathrm{n}$-th bus as a reference for the equations of buses written as Eq. (29).

$$
\left[\begin{array}{l}
V_{1 n} \\
V_{2 n} \\
V_{3 n} \\
V_{4 n}
\end{array}\right]=\left[\begin{array}{llll}
Z_{11} & Z_{12} & Z_{13} & Z_{14} \\
Z_{21} & Z_{22} & Z_{23} & Z_{24} \\
Z_{31} & Z_{32} & Z_{33} & Z_{34} \\
Z_{41} & Z_{42} & Z_{43} & Z_{44}
\end{array}\right] \cdot\left[\begin{array}{c}
I_{1} \\
I_{2} \\
I_{3} \\
I_{4}
\end{array}\right]
$$

The first line translation of Eq. (29) is obtained as Eq. (30).

$$
V_{1 n}=Z_{11} \cdot I_{1}+Z_{12} \cdot I_{2}+Z_{13} \cdot I_{3}+Z_{41} \cdot I_{4}
$$

The substitution is for $I_{3}=d_{3} I_{D}$ and $I_{4}=d_{4} I_{D}$ in Eq. (2), then completion of the equation simultaneously to be $I_{D}$ is produced as Eq. (31).

$$
\begin{gathered}
I_{D}=\frac{-Z_{11}}{d_{3} \cdot Z_{11}+d_{4} \cdot Z_{14}} \cdot I_{1}+\frac{-Z_{12}}{d_{3} \cdot Z_{13}+d_{4} \cdot Z_{14}} \cdot I_{2} \\
+\frac{-Z_{11}}{d_{3} \cdot Z_{13}+d_{4} \cdot Z_{14}} \cdot I_{n}^{0}
\end{gathered}
$$

where, $I_{n}^{0}$ is no-load current and identified as a value like Eq. (32).

$$
I_{n}^{0}=-\frac{V_{1 n}}{Z_{11}}
$$

For explanation help it is mentioned, that $I^{0}{ }_{n}$ is called a noload current. Schematic diagram for the explanation of the noload current $I_{n}^{0}$ is shown in Figure 2.

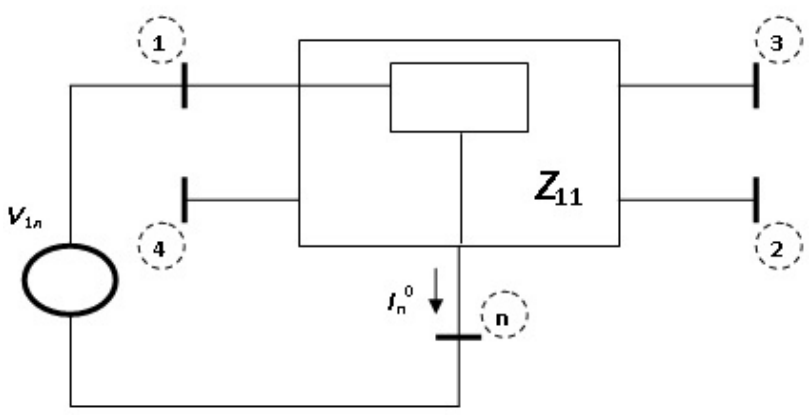

Figure 2. Schematic diagram for explanation of no-load current $I^{0}{ }_{n}$

Based on Figure 2, when all loads and generating units are released from the voltage system $V_{I n}$ is used in bus-1, only the current $I_{n}^{0}$ will flow through the shunt connection to the n-th bus. This current is generally small and relatively constant.

The simplification coefficient is found in Eq. (31) by Eq. (32) is obtained as Eq. (33).

$$
t_{Z 1}=\frac{Z_{11}}{d_{3} \cdot Z_{11}+d_{4} \cdot Z_{14}} \text { and } t_{Z 2}=\frac{Z_{12}}{d_{3} \cdot Z_{11}+d_{4} \cdot Z_{14}}
$$

then Eq. (32) becomes as Eq. (34).

$$
I_{D}=-t_{Z 1} \cdot I_{1}-t_{Z 2} \cdot I_{2}-t_{Z 1} \cdot I_{n}^{0}
$$

The substitution of Eq. (34) to Eq. (2) is obtained as Eq. (35) and (36).

$$
I_{3}=-d_{3} \cdot t_{Z 1} \cdot I_{1}-d_{3} \cdot t_{Z 2} \cdot I_{2}-d_{3} \cdot t_{Z 1} \cdot I_{n}^{0}
$$

and

$$
I_{4}=-d_{4} \cdot t_{Z 1} \cdot I_{1}-d_{4} \cdot t_{Z 2} \cdot I_{2}-d_{4} \cdot t_{Z 1} \cdot I_{n}^{0}
$$

Furthermore, we can change the Eqns. (35) and (36) with the definition of the transformation of the currents $I_{1}, I_{2}, I_{3}$ and $I_{4}$ is shown in Eq. (30), into new currents, $I_{1}, I_{2}$ and $I_{n}^{0}$, then is obtained as Eq. (37).

$$
\begin{gathered}
{\left[\begin{array}{l}
V_{1 n} \\
V_{2 n} \\
V_{3 n} \\
V_{4 n}
\end{array}\right]=\left[\begin{array}{ccc}
1 & 0 & 0 \\
0 & 1 & 0 \\
-d_{3} \cdot t_{Z 1} & -d_{3} \cdot t_{Z 2} & -d_{3} \cdot t_{Z 1} \\
-d_{4} \cdot t_{Z 1} & -d_{4} \cdot t_{Z 2} & -d_{4} \cdot t_{Z 1}
\end{array}\right] \cdot\left[\begin{array}{l}
I_{1} \\
I_{2} \\
I_{n}^{0}
\end{array}\right]} \\
=C \cdot\left[\begin{array}{l}
I_{1} \\
I_{2} \\
I_{n}^{0}
\end{array}\right]
\end{gathered}
$$

Eq. (37) can be written as Eq. (38).

$$
P_{\text {Losses }}=\left[\begin{array}{lll}
I_{1} & I_{2} & I_{n}^{0}
\end{array}\right] \cdot C^{T} \cdot R_{\text {bus }} \cdot C^{*} \cdot\left[\begin{array}{c}
I_{1} \\
I_{2} \\
I_{n}^{0}
\end{array}\right]
$$

Against each bus the generator is assumed, that $Q_{G i}$ (reactive power) is a very small constant of $S_{i}$ from $P_{G i}$ (active power) at each review period. It is equivalent to assuming, that each of the generators operates at a constant $\cos \varphi$ (power factor) over the same period. Therefore, it can be written as Eq. (39),

$$
P_{G i}+j Q_{G i}=\left(1+j S_{i}\right) \cdot P_{G i}
$$

where,

$$
S_{i}=\frac{Q_{G i}}{P_{G i}}=\text { real amount. }
$$

The generator output current is given by Eq. (40).

$$
I_{i}=\frac{\left(1+j S_{i}\right)}{V_{i}^{*}} \cdot P_{G i}=\alpha_{i} \cdot P_{G i}
$$

Using Eq. (40) to calculate the current on two generators is obtained:

$$
\begin{aligned}
& I_{1}=\frac{\left(1+j S_{1}\right)}{V_{1}^{*}} \cdot P_{G 1}=\alpha_{1} \cdot P_{G 1} \\
& I_{2}=\frac{\left(1+j S_{2}\right)}{V_{2}^{*}} \cdot P_{G 2}=\alpha_{2} \cdot P_{G 2}
\end{aligned}
$$

Guided by Eqns. (40), values of currents and $I_{1}, I_{2}$ and $I^{0}{ }_{n}$ can be written in matrix form as Eq. (41).

$$
\left[\begin{array}{c}
I_{1} \\
I_{2} \\
I_{n}^{0}
\end{array}\right]=\left[\begin{array}{ccc}
\alpha_{1} & 0 & 0 \\
0 & \alpha_{2} & 0 \\
0 & 0 & I_{n}^{0}
\end{array}\right] \cdot\left[\begin{array}{c}
P_{G 1} \\
P_{G 2} \\
1
\end{array}\right]
$$
(42).

The substitution of Eq. (41) to Eq. (38) is obtained as Eq.

$$
P_{\text {Losses }}=\left[\begin{array}{c}
P_{G 1} \\
P_{G 2} \\
1
\end{array}\right]^{T} \cdot\left[\begin{array}{ccc}
\alpha_{1} & 0 & 0 \\
0 & \alpha_{2} & 0 \\
0 & 0 & I_{n}^{0}
\end{array}\right] \cdot C^{T} \cdot R_{\text {bus }} \cdot C^{*} \cdot\left[\begin{array}{c}
P_{G 1} \\
P_{G 2} \\
1
\end{array}\right]
$$


Assumes that there are 3 (three) matrices, namely $D, E$, and $F$, hence $(D E F)^{T}=D^{T} \cdot E^{T} \cdot F^{T}$, and taken complex conjugate on each side giving $(D E F)^{T}=D^{T} E^{T} F^{T}$. It can be shown that the matrix $T_{\alpha}$ in Eq. (42) has the same simple form as the complex conjugate of its transpose. The matrix with the characteristics referred to is called the Hermitian matrix. Each of the $m_{i j}$ closely diagonal elements of the Hermitian matrix is the conjugate complex of the element $m_{i j}$, and all the diagonal elements are real. Consequently, adding $T_{\alpha}$ to $T_{\alpha}^{*}$ it will remove the closest elemental part of the diagonal and obtain a symmetrical real part of the matrix $T_{\alpha}$ that defined as Eq. (43).

$$
\begin{array}{ccc}
L_{11} & L_{12} & L_{20} / 2 \\
L_{21} & L_{22} & L_{20} / 2 \\
\left\lfloor L_{10} / 2\right. & L_{20} / 2 & \left.L_{00}\right\rfloor
\end{array}=\frac{T_{\alpha}+T_{\alpha}^{*}}{2}
$$

The addition of Eq. (42) to a complex conjugate using Eq. (43) is produced as Eq. (44)

$$
\begin{gathered}
\left.P_{\text {Losses }}=\left[\begin{array}{lllll}
L_{11} & L_{12} & L_{20} / 2 \\
P_{G 2} & 1
\end{array}\right] \cdot \begin{array}{ccc}
L_{21} & L_{22} & L_{20} / 2 \\
\left\lfloor L_{10} / 2\right. & L_{20} / 2 & L_{00}
\end{array}\right] \\
{\left[\begin{array}{c}
P_{G 1} \\
P_{G 2} \\
1
\end{array}\right]}
\end{gathered}
$$

where, $L_{12}=L_{21}$.

Further description of the Eq. (44) by multiplication of rowcolumn gives:

$$
\begin{gathered}
P_{\text {Losses }}=L_{11} \cdot P_{G 1}+2 \cdot L_{12} \cdot P_{G 1} \cdot P_{G 2}+L_{22} \cdot P_{G 2}+ \\
L_{10} \cdot P_{G 1}+L_{20} \cdot P_{G 2}+L_{00}
\end{gathered}
$$

or as Eq. (45).

$$
\begin{gathered}
P_{\text {Losses }}=\sum_{i=1}^{2} \sum_{j=1}^{2} P_{G i} \cdot L_{i j} \cdot P_{G j}+\sum_{i=1}^{2} L_{i 0} \cdot P_{G i}+ \\
L_{00}
\end{gathered}
$$

Arrangement in the form of matrix is obtained as Eq. (46).

$$
\begin{aligned}
P_{\text {Losses }} & =\left[\begin{array}{ll}
P_{G 1} & P_{G 2}
\end{array}\right] \cdot\left[\begin{array}{ll}
L_{11} & L_{12} \\
L_{21} & L_{22}
\end{array}\right] \cdot\left[\begin{array}{l}
P_{G 1} \\
P_{G 2}
\end{array}\right] \\
& +\left[\begin{array}{ll}
P_{G 1} & P_{G 2}
\end{array}\right] \cdot\left[\begin{array}{l}
B_{10} \\
B_{20}
\end{array}\right]+B_{00}
\end{aligned}
$$

or in a general form as Eq. (47) as the Kron's equation.

$$
P_{\text {Losses }}=P_{G}^{T} \cdot L \cdot P_{G}+P_{G}^{T} \cdot L_{0}+L_{00}
$$

If the system has a source, the vector and matrix of Eq. (47) will have $k$ rows or $k$ columns and will generally result in the power loss on transmission line equations as follows in Eq. (48).

$$
P_{\text {Losses }}=\sum_{i=1}^{k} \sum_{j=1}^{k} P_{G i} \cdot L_{i j} \cdot P_{G j}+\sum_{i=1}^{k} L_{i 0} \cdot P_{G i}+L_{00}
$$

\section{CONCLUSIONS}

Based on the results and discussion, then drawn the conclusion according to the purpose of the theoretical review. Minimization cost generation method without covering the power loss on transmission line will be a failure, even if the incremental cost for fuel is lower than other generating units, but the location far from the load centers resulted in the power loss, so the transmission line value is higher. Coordination equation is an equation with an incremental cost for fuel and power line loss losses. In the coordination equation, the cost of generation is economical and is achieved when the incremental cost for the total fuel is equal to the value of the penalty for all generating units. To calculate the achievement of economic dispatching value in electric power system with type of thermal generation with calculation of power loss of transmission line and value of limitation of generation of generating unit as generating function, hence can be reached by some way of calculation.

\section{REFERENCES}

[1] Elgerd, O.I. (1971). Electric Energy Systems Theory: An Introduction. Tata McGraw-Hill Publishing Company Ltd., New Delhi, 274-311.

[2] Grainger, J.J., Stevenson, W.D. (1994). Power System Analysis. McGraw-Hill, Inc., New York, 531-590.

[3] Wood A.J., Wollengerg, B.F. (1996). Power Generation, Operation, and Control. 2nd ed. John Wiley \& Sons, Inc., New York, 111-123.

[4] Saadat, H. (1999). Power System Analysis, McGraw-Hill Companies, Inc., Boston, 257-313.

[5] Das, D. (2006). Electrical Power Systems. New Age International Limited, New Delhi, 405-446.

[6] Teh, J. (2016). Analysis of dynamic thermal rating system of transmission lines. Ph.D. dissertation. The University of Manchester, Manchester, England.

[7] Kirschen, D.S., Strbac, G. (2004). Fundamentals of Power System Economics. John Wiley \& Sons, Ltd., Chichester, West Sussex (England), 148-199.

[8] Breeze, P. (2010). The Cost of Power Generation, Business Insight, Ltd., Warwick, Warwickshare, 20-46.

[9] Sonmez, Y.M. (2013). Estimation of fuel cost curve parameters for thermal power plants using the ABC algorithm. TUBITAK: Turkish Journal Electrical Engineering \& Computer Sciences, 21: 1827-1841. https://doi.org/10.3906/elk-1203-10

[10] Low, S.H. (2015). Optimal power flow for future smart grid. Clean Energy Institute, University of Washington. https://www.cei.washington.edu/seminars/optimalpower-flow-for-future-smart-grid/, accessed on Jan. 30, 2017.

[11] Khan, M.J., Kumar, Y. (2016). Optimal power flow (OPF) Formulation and reactive power optimization of power systems using conventional optimization technique (interior point method). International Journal of Mathematics Research, 8(3): 251-263.

[12] Kirschen, D. (2010). New formulations of the optimal power flow problem. University of Manchester. https://www.newton.ac.uk/files/seminar/201005251545 17001-152377.pdf, accessed on Jan 30, 2017.

[13] Ebeed, M., Kamel, S., Jurado, F. (2018). Classical and recent aspects of power system optimization: Optimal 
power flow using recent optimization techniques. Classical and Recent Aspects of Power System Optimization, 157-183. https://doi.org/10.1016/B978-012-812441-3.00007-0

\section{NOMENCLATURE}

$I_{1} \quad$ currents at the generating bus (1-st bus)

$I_{2} \quad$ currents at the generating bus (2-nd bus)

$I_{2} \quad$ currents at the load bus (3-rd bus)

$I_{4} \quad$ currents at the load bus (4-th bus)

$I_{n}^{0} \quad$ no-load current, will flow through the shunt connection to the $\mathrm{n}$-th bus (neitral point)

$I_{D} \quad$ currents injected at all of the load bus

$d_{3}$

$d_{4}$

$P_{\text {Losses }}$

$P_{\text {Demand }}$

$L F$

$P_{G i}$

$P_{G 1}$

$P_{G 2}$

$P_{G T}$

$i$

$k$

$Q_{G i}$

$S_{G i}$

$C_{i}$

$C_{1}$

$C_{2}$

$\lambda$ percentage of currents injected at the load bus (3-rd bus)

percentage of currents injected at the load bus (4-th bus)

power losses on the transmission line

load of the system

Lagrange function

the real power output from the $i$-th generating unit the real power output of the 1-st generating unit the real power output of the 2-nd generating unit the total real power output of the generating unit number

number or number of digits

the reactive power output from the $i$-th generating units units, $S_{i}$

cost of fuel of the $i$-th generating unit

cost of fuel of the 1-st generating unit

cost of fuel of the 2-nd generating unit

incremental cost of the fuel (in the Langrange Multifier) the apparent power output from the $i$-th generating

the value of $\lambda$ of $i$-th bus, or the $i$-th bus penalty

$\lambda_{i}$

$\lambda_{1}$

$\lambda_{2}$

$a_{1}$

$b_{1}$

$a_{T}$

$b_{T}$

$L_{11}$

$L_{12}$

$L_{10}$

$L_{21}$

$L_{22}$

$L_{20}$

$P_{G i}^{\min }$

$P_{G i}^{\max }$

$V_{1 n}$

$L_{i j}$

$L_{i 0}$

$L_{00}$

\section{Greek symbols}

$\Delta$

$d$

$\delta$

$\Sigma$

K the change in of the variable the derivative of single variable the derivative of two or more variables sum the cost of a penalty factor for all generators 